\title{
DDR2 facilitates hepatocellular carcinoma invasion and metastasis via activating ERK signaling and stabilizing SNAIL1
}

Binhui Xie ${ }^{1 \dagger}$, Weihao Lin ${ }^{2 \dagger}$, Junming Ye ${ }^{3}$, Xiaonong Wang ${ }^{1}$, Bing Zhang ${ }^{4}$, Shiqiu Xiong ${ }^{5}$, Heping Li $i^{4^{*}}$ and Guosheng $\operatorname{Tan}^{4^{*}}$

\begin{abstract}
Background: Several studies have found that DDR2 is up-regulated in many tumor types and facilitates tumor progression. However, the role of DDR2 in hepatocellular carcinoma (HCC) progression and its downstream signaling pathways remain unclear.

Methods: DDR2 expression was assessed in several cell lines and 112 pairs of HCC and matched adjacent noncancerous liver tissues. Clinical significance of DDR2 in HCC was analyzed. Phosphorylated DDR2 (p-DDR2) expression was detected by immunoblotting to evaluate its correlation with DDR2. The effect of DDR2 on HCC cell migration and invasion were examined. Cycloheximide chase experiments were performed to detect the half-life of SNAIL1. Moreover, DDR2 expression was detected by immunohistochemistry to evaluate its correlation with SNAIL1. The regulatory effect of DDR2 on ERK signaling, SNAIL1, EMT, MT1-MMP and MMP2 was confirmed by immunoblotting. The effect of type I collagen on DDR2/ERK2/SNAIL1 signaling was assessed.
\end{abstract}

Results: DDR2 was more highly expressed in HCC than in non-HCC tissues. DDR2 overexpression was correlated with clinicopathological features of poor prognosis. Clinical analysis revealed that DDR2 is an independent prognostic marker for predicting overall survival and disease free survival of HCC patients. Overexpression of DDR2 is associated with p-DDR2 amplification. In vitro studies showed that DDR2 facilitates HCC cell invasion, migration and EMT via activating ERK2 and stabilizing SNAIL1. DDR2 can up-regulate MT1-MMP and MMP2 expression through ERK2/SNAIL1 signaling in HCC. Additionally, collagen I can induce DDR2/ERK2/SNAIL1 signaling activation in HCC cells.

Conclusions: Our findings suggest that DDR2 plays an important role in promoting HCC cell invasion and migration, and may serve as a novel therapeutic target in HCC.

Keywords: DDR2, HCC, ERK, SNAIL1, EMT

\section{Background}

Hepatocellular carcinoma (HCC) is one of the most prevalent malignant tumors worldwide and is the second leading cause of cancer-related death in China [1-3]. In recent years, the incidence of $\mathrm{HCC}$ is increasing in many parts of the world including the United States, partly due to the rise in hepatitis $C$ virus infection [4-6]. Hepatic

\footnotetext{
*Correspondence: lihp1979@163.com; tgs918@163.com

${ }^{\dagger}$ Equal contributors

${ }^{4}$ Department of Medical Imaging, The First Affiliated Hospital of Sun Yat-sen University, Guangzhou 510080, China

Full list of author information is available at the end of the article
}

resection offers the chance of a cure for patients with HCC, but the prognosis remains very poor $[7,8]$. Hence, understanding the molecular mechanisms involved in hepatocarcinogenesis and exploring prognostic markers and therapeutic targets of HCC is urgently needed.

The discoidin domain receptors (DDRs), consisting of DDR1 and DDR2, are distinctive receptor tyrosine kinases (RTKs) and signal in response to collagens instead of soluble peptide growth factors [9-11]. Multiple studies have suggested that DDR2 can regulate cell adhesion, migration and matrix remodeling [12, 13]. DDR2 have also been shown to exhibit aberrant expression patterns 
in several tumor types, including nasopharyngeal and prostate cancer [14-16]. Furthermore, it was found that the abnormal expression of DDR2 implicated in cancer progression and a poor prognosis [17, 18]. However, the role of DDR2 in HCC and the molecular mechanisms by which DDR2 exerts its biological function are still unclear.

SNAIL1, a member of transcription factors, is critical for inducing and sustaining cancer epithelial-mesenchymal transition (EMT) [19, 20]. SNAIL1-induced EMT has been demonstrated essential for promoting tumor cell migration and invasion in multiple tumor types [21, 22]. Recently, Zhang et al. have found that SNAIL1 is regulated by the DDR2/ERK2 signaling pathway in breast cancer [23]. In the previous studies, ERK signaling pathway has been considered to promote the progression of HCC $[24,25]$. It is also known that SNAIL1 plays an essential role in regulating HCC EMT [26, 27]. However, whether DDR2 is implicated in regulating the ERK signaling and SNAIL1 in HCC has not been clarified.

As representative epithelial-specific and mesenchymalspecific biomarkers, E-cadherin and Vimentin are crucial factors in the invasion, migration and EMT of HCC [21]. Down-regulation of E-cadherin and up-regulation of Vimentin are used as biomarkers indicating an epithelial cell has undergone EMT in HCC [21]. Multiple studies have revealed that EMT associated with patients poor prognosis is an important step in the invasion and migration of HCC $[21,26]$. Importantly, SNAIL1-induced E-cadherin down-regulation and Vimentin up-regulation have been demonstrated essential for triggering the EMT of HCC [27]. However, whether DDR2 is implicated in regulating the expression of E-cadherin and Vimentin in HCC are still unknown.

In this study, we found that DDR2 was more highly expressed in HCC tissues than that in non-tumor tissues, and DDR2 overexpression was correlated with poor clinicopathological features and outcome of HCC patients. Our study demonstrated that DDR2 has an oncogenic role in HCC tumorigenesis by facilitating cancer cell invasion, migration and epithelial-mesenchymal transition via activating ERK signaling and stabilizing SNAIL1. Additionally, DDR2 can up-regulate membrane type-1 matrix metalloproteinase (MT1-MMP) and MMP2 expression through ERK2/SNAIL1 signaling in HCC. Our study identified that DDR2 is a novel regulator of EMT through stabilizing SNAIL1, indicating its potential therapeutic value for reducing $\mathrm{HCC}$ invasion and metastasis.

\section{Materials and methods}

\section{Tissue samples}

A total of 112 pairs of HCC and corresponding adjacent non-tumorous liver tissues $(>2.0 \mathrm{~cm}$ from the resection margin) were obtained from patients undergoing hepatectomy between February 2007 and December 2009 at the Department of Hepatobiliary Surgery, the First Affiliated Hospital of Gannan Medical University. The patient characteristics are shown in Table 1. All patients provided written informed consent before hepatectomy, and our study were approved by the Ethics Committee of the First Affiliated Hospital of Gannan Medical University according to the 1975 Declaration of Helsinki.

Table 1 Correlations between DDR2 expression and clinicopathologic features in HCC

\begin{tabular}{|c|c|c|c|c|}
\hline \multirow[t]{2}{*}{ Characteristics } & \multirow[t]{2}{*}{$\mathrm{n}$} & \multicolumn{2}{|c|}{ DDR2 expression } & \multirow[t]{2}{*}{$P$ value } \\
\hline & & High & Low & \\
\hline \multicolumn{5}{|l|}{ Gender } \\
\hline Female & 25 & 15 & 10 & \\
\hline Male & 87 & 41 & 46 & 0.364 \\
\hline \multicolumn{5}{|l|}{ Age (year) } \\
\hline$\leq 45$ & 29 & 10 & 19 & \\
\hline$>45$ & 83 & 46 & 37 & 0.083 \\
\hline \multicolumn{5}{|l|}{ HBsAg status ${ }^{a}$} \\
\hline Negative & 22 & 7 & 15 & \\
\hline Positive & 90 & 49 & 41 & 0.095 \\
\hline \multicolumn{5}{|l|}{ Cirrhosis } \\
\hline No & 37 & 15 & 22 & \\
\hline Yes & 75 & 41 & 34 & 0.228 \\
\hline \multicolumn{5}{|l|}{$\operatorname{AFP}(\mu \mathrm{g} /)^{\mathrm{b}}$} \\
\hline$\leq 400$ & 55 & 23 & 32 & \\
\hline$>400$ & 57 & 33 & 24 & 0.130 \\
\hline \multicolumn{5}{|l|}{ Tumor size } \\
\hline$\leq 5 \mathrm{~cm}$ & 37 & 14 & 23 & \\
\hline$>5 \mathrm{~cm}$ & 75 & 42 & 33 & 0.107 \\
\hline \multicolumn{5}{|l|}{ Tumor number } \\
\hline Single & 86 & 37 & 49 & \\
\hline Multiple & 26 & 19 & 7 & $0.013^{*}$ \\
\hline \multicolumn{5}{|l|}{ Tumor capsule } \\
\hline Complete & 20 & 7 & 13 & \\
\hline Incomplete & 92 & 49 & 43 & 0.217 \\
\hline \multicolumn{5}{|c|}{ Vascular invasion } \\
\hline No & 86 & 36 & 50 & \\
\hline Yes & 26 & 20 & 6 & $0.003^{*}$ \\
\hline \multicolumn{5}{|c|}{ Edmondson grade } \\
\hline$|/| \mid$ & 64 & 24 & 40 & \\
\hline III/IV & 48 & 32 & 16 & $0.004^{*}$ \\
\hline \multicolumn{5}{|l|}{ TNM stage } \\
\hline$|/| \mid$ & 67 & 25 & 42 & \\
\hline III/IV & 45 & 31 & 14 & $0.002^{*}$ \\
\hline
\end{tabular}

${ }^{\mathrm{a}} \mathrm{HbsAg}$ hepatitis B surface antigen, ${ }^{\mathrm{b}} \mathrm{AFP}$ a-fetoprotein, ${ }^{*} P<0.05$ 


\section{Cell lines}

The immortalized normal human liver cell line L02 and five HCC cell lines including SMMC-7721, Huh-7, HepG2, Hep3B and MHCC-97H were obtained from from the Type Culture Collection of the Chinese Academy of Sciences (Shanghai, China). All cell lines were cultured in Dulbecco's modified Eagle medium (DMEM, Gibco, Grand Island, NY, USA) containing $10 \%$ fetal bovine serum (FBS, Gibco).

\section{Quantitative reverse transcription-polymerase chain reaction (qRT-PCR)}

Total RNA was extracted using TRIzol reagent (Invitrogen, USA). qRT-PCR was done in an ABI 7500 system using the SYBR $^{\odot}$ Premix Ex Taq ${ }^{\mathrm{Tm}}$ II (Tli RNaseH Plus) (TakaRa, Japan). DDR2 primers: forward 5'-CTCCCAGAATTTGC TCCAG-3'; reverse 5'-GCCACATCTTTTCCTGAGA-3-3'. SNAIL1 primers: forward 5'- GCT CCACAAGCACCAAG AGT-3'; reverse 5'- ATTCCATGGCAGTGAGAAGG-3'. $\beta$-actin primers: forward 5'-GGGAA ATCGTGCGTGAC AT-3'; reverse 5'-C TGGA AGGTGGACAGCGAG-3'.

\section{Western immunoblotting}

The following primary antibodies were used in the western immunoblotting assays: SNAIL1 (ab180714, Abcam, Cambridge, UK), Vimentin (ab137321, Abcam, Cambridge, UK), E-cadherin (ab15148, Abcam, Cambridge, UK), MT1MMP (ab53712, Abcam, Cambridge, UK), DDR2 (sc-8989, Santa Cruz, CA, USA), p-DDR2 (MAB25382, R\&D systems Inc, USA), ERK1/2 (sc-292838, Santa Cruz, CA, USA), p-ERK1/2 (sc-101760, Santa Cruz, CA, USA), MMP2 (sc-10736, Santa Cruz, CA, USA) and $\beta$-actin polyclonal antibody (sc-130656, Santa Cruz, CA, USA). Immunoblotting assays were performed as reported previously [3].

\section{Immunohistochemical staining}

Immunohistochemical assay was performed on paraformaldehyde-fixed paraffin sections as previous reported [28]. The DDR2 (sc-8989, Santa Cruz, CA, USA) and SNAIL1 (ab180714, Abcam, Cambridge, UK) primary antibodies were used at a 1:100 dilution in the immunohistochemistry assays. The immunostaining intensity and average percentage of positive cells were evaluated as previous reported [28]. Immunostaining intensity was evaluated as four grades: 0 , negative; 1 , weak; 2 , moderate; 3 , strong. And the percentage of positive cells was categorized as the following grades: $0,0 \% ; 1,1$ to $10 \% ; 2,11$ to $50 \%$; 3,51 to $80 \%$; and $4>80 \%$. The immunostaining intensity and average percentage of positive cells were evaluated for ten independent high magnification fields. By multiplying the staining intensity and the percentage of positive cells, the final weighed expression score was obtained (0-12).

\section{Expressing plasmids and RNAi transfection}

The DDR2 expressing plasmid and its empty plasmid pCMV6-XL6 were both obtained from Origene Technologies Inc. (Rockville, USA). DDR2 expressing plasmid or empty plasmid pCMV6-XL6 was transfected into Hep3B cells using TurboFectin Transfection Reagent purchased from Origene Technologies Inc. (Rockville, USA). The DDR2 shRNA and scrambled shRNA vector pRS were also purchased from Origene Technologies Inc. (Rockville, USA). DDR2 unique 29mer shRNA constructs in pRS Vector or scrambled shRNA vector pRS was transfected into MHCC-97H cells using TurboFectin Transfection Reagent purchased from Origene Technologies Inc. (Rockville, USA). The specific siRNA sequences against ERK2 (sc-35335), against SNAIL1 (sc-38398) and the scramble siRNAs (sc-37007) were obtained from Santa Cruz Biotechnology (Santa Cruz, USA).

\section{Cycloheximide (CHX) chase experiments}

To assess SNAIL1 protein stability, HCC cells were treated with $50 \mathrm{mg} / \mathrm{ml}$ cycloheximide (final concentration, Sigma) following expressing plasmids or RNAi transfection for $72 \mathrm{~h}$. At the given time points, HCC cells were harvested, flash-frozen, and analyzed by Western blotting as described above. SNAIL1 protein degradation rates were quantified by densitometry using time point zero as $100 \%$.

\section{Co-Immunoprecipitation assay}

Total protein lysate was obtained in immunoprecipitation buffer. The lysate was precleared with protein A/G-agarose beads. Total protein in supernatants was qualified by BCA method. Then, total protein was diluted into $1 \mu \mathrm{g} / \mu \mathrm{L}$ with PBS and mixed with primary antibodies against DDR2, SNAIL1, ERK2 or IgG. The mixtures were shaken on rotating shaker for overnight at $4{ }^{\circ} \mathrm{C}$. The supernatant was obtained and used in the immunoblotting assay.

\section{Transwell migration and invasion assays}

Transwell migration assays were performed in 12 well plates with $8-\mu \mathrm{m}$ BioCoat control inserts (Bedford, MA). HCC cells that were suspended in serum-free medium were added into the upper chamber and medium with $10 \%$ FBS was added into the lower chamber. The cells on the inner layer were softly removed and the adherent tumor cells on undersurface of the insert were stained with crystal violet. Transwell invasion assays were performed in BioCoat Matrigel invasion chamber containing an $8 \mu \mathrm{m}$ pore-size PET membrane with a uniform layer of $\mathrm{BD}$ Matrigel basement membrane matrix (Becton Dickinson Labware). The following protocols were the same as cell migration assays. 


\section{Statistical analysis}

Data were showed as the Mean \pm SEM. Statistical significance was established, with the SPSS 16.0 statistical software package (SPSS Inc., Chicago, IL), using a two-tailed Student's $t$ test, a Kaplan-Meier plot, a log-rank test, a Spearman correlation coefficient analysis, a Chi-square test or Fisher's exact test. Independent prognostic factors were assessed by the Cox proportional hazards stepwise regression model. $\mathrm{P}$ values were 2 -sided and a $P$ value of less than 0.05 was considered statistical significance.

\section{Results}

\section{Up-regulation of DDR2 is a frequent event in HCC lines}

We first analyzed DDR2 expression in an immortalized non-tumourigenic hepatocyte cell line L02 and a panel of HCC cell lines (SMMC-7721, Huh-7, HepG2, Hep3B and $\mathrm{MHCC}-97 \mathrm{H})$ using qRT-PCR and Western blotting. We found that DDR2 expression was evidently upregulated in all HCC cell lines as compared with that in a non-tumourigenic hepatocyte cell line L02 $(P<0.01$, respectively, Fig. 1a-b). Furthermore, DDR2 expression in the highly invasive $\mathrm{HCC}$ cell line (MHCC-97H) were evidently higher than those in the low invasive $\mathrm{HCC}$ cell lines including SMMC-7721, Huh-7, HepG2, Hep3B $(P<0.01$, respectively, Fig. 1a-b). The characteristics of each cell line are shown in Table 2. Our data indicates that elevated DDR2 expression confers increased invasive potential of HCC cells. In our study, MHCC-97H expressed the highest expression level of DDR2 in HCC cell lines and the Hep3B expressed the lowest expression level of DDR2 among the five HCC cell lines. Thereby, Hep3B cell line was selected for DDR2 overexpression experiment here, while MHCC-97H cell line were used in DDDR2 knockdown experiment.

\section{Clinical significance of DDR2 expression in HCC tissues}

To analyze the clinical significance of DDR2 in HCC, we detected the DDR2 expression in 112 pairs of HCC and matched tumor-adjacent samples using qRT-PCR and Western blotting, and found that DDR2 expression was prominently higher in the HCC samples than that in the noncancerous samples $(P<0.01$, respectively, Fig. $1 c-d)$. In our study, we also detected the phosphorylated DDR2 (p-DDR2) expression in HCC, which was previously shown to be associated with DDR2 activation. We found that p-DDR2 expression level was evidently higher in the HCC samples than that in the noncancerous samples $(P<0.01$, Fig. $1 d)$. To further identify the clinical role of DDR2 in HCC, we detected the correlations of the DDR2 protein expression with clinicopathological characteristics,
A

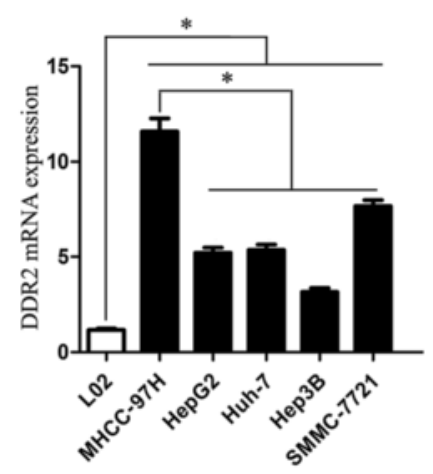

C

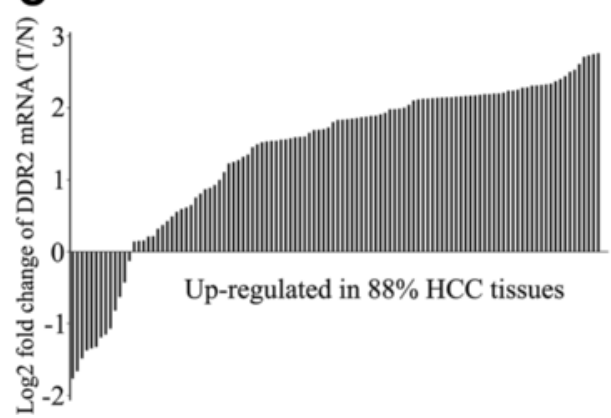

B

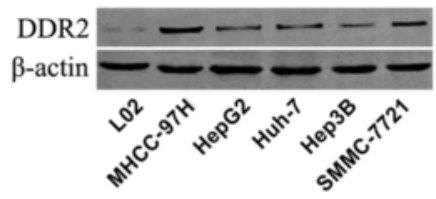

D

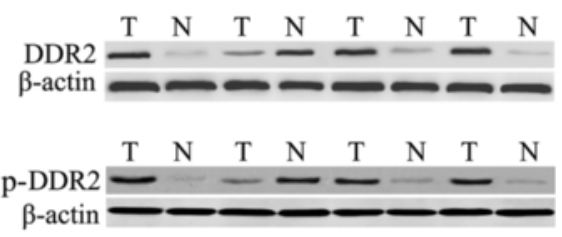

Fig. 1 The expression of DDR2 in cell lines and HCC tissues. a DDR2 mRNA expression in the immortalized normal human liver cell line L-02 and five hepatoma cell lines (MHCC-97H, HepG2, Huh-7, Hep3B and SMMC-7721) using qRT-PCR $\left(n=3,{ }^{*} P<0.01\right)$. b DDR2 protein expression in the immortalized normal human liver cell line L-02 and five hepatoma cell lines (MHCC-97H, HepG2, Huh-7, Hep3B and SMMC-7721) using immunoblotting $(n=3, P<0.01)$. c DDR2 mRNA expression in HCC tissues (T) and corresponding adjacent no tumorous tissues ( $N$ ) using qRT-PCR $(n=112, P<0.01)$. $\mathbf{d}$ The protein expression of DDR2 and phosphorylated DDR2 ( $p$-DDR2) in HCC tissues (T) and corresponding adjacent no tumorous tissues $(\mathrm{N})$ using immunoblotting $(n=112, P<0.01$, respectively) 
Table $\mathbf{2}$ Characteristics of the cell lines used in this study

\begin{tabular}{llllll}
\hline Cell line & Type & Characteristics & & \\
\cline { 3 - 5 } & & HBsAg $^{\mathrm{a}}$ & P53 & AFP & Invasion \& migration \\
\hline L02 & Liver cell line & Negative & Wild type p53 & Negative & No \\
Hep3B & Hepatoma cell line & Positive & P53 deletion & Positive & Low \\
HepG2 & Hepatoma cell line & Negative & Wild type p53 & Positive & Low \\
Huh-7 & Hepatoma cell line & Negative & P53 mutation & Positive & Low \\
SMMC-7721 & Hepatoma cell line & Negative & P53 mutation & Positive & Low \\
MHCC-97H & Hepatoma cell line & Positive & P53 mutation & Positive & High \\
\hline
\end{tabular}

${ }^{\mathrm{a}} \mathrm{Hbs} \mathrm{Ag}$ hepatitis B surface antigen, ${ }^{\mathrm{b}} \mathrm{AFP}$ a-fetoprotein

including gender, age, HBsAg status, AFP level, tumor size, liver cirrhosis, capsule formation, Edmondson-Steiner grade, tumor number, vascular invasion and TNM stage. In this study, the median DDR2 protein expression level was used as the cutoff point to divide into low-expressing and high expressing groups. Strikingly, DDR2 expression was evidently correlated with tumor number, vascular invasion, Edmondson-Steiner grade and TNM stage (Table 1). However, no relationship was found between the DDR2 expression and other clinicopathological characteristics including gender, age, HBsAg status, AFP level, tumor size, liver cirrhosis, capsule formation (Table 1).

\section{DDR2 expression is an independent prognostic factor for} HCC

In this study, the median DDR2 protein expression was used as the cutoff point to divide into high-expressing and low-expressing groups for HCC patients' survival. HCC patients in the high DDR2-expressing group had obviously reduced overall survival and disease-free survival. The 5-year overall survival rate of the high DDR2-expressing group was significantly lower than that of the low DDR2-expressing group $(P<0.01)$ (Fig. 2a). The 5-year disease-free survival rate of the high DDR2expressing group was obviously lower than that of the low DDR2-expressing group $(P<0.01)$ (Fig. $2 b)$. In our study, we also performed Univariate analysis and Cox proportional hazards regression analysis. The univariate analysis revealed that DDR2 level, HBsAg status, tumor number, vascular invasion, Edmondson-Steiner grade and TNM stage were the prognosis factors for HCC patients (Table 3). In a multivariate analysis model, DDR2 expression level was obviously associated with overall survival (HR 2.169; $95 \%$ CI, 1.198-3.927; $P<0.05)$ and disease-free survival
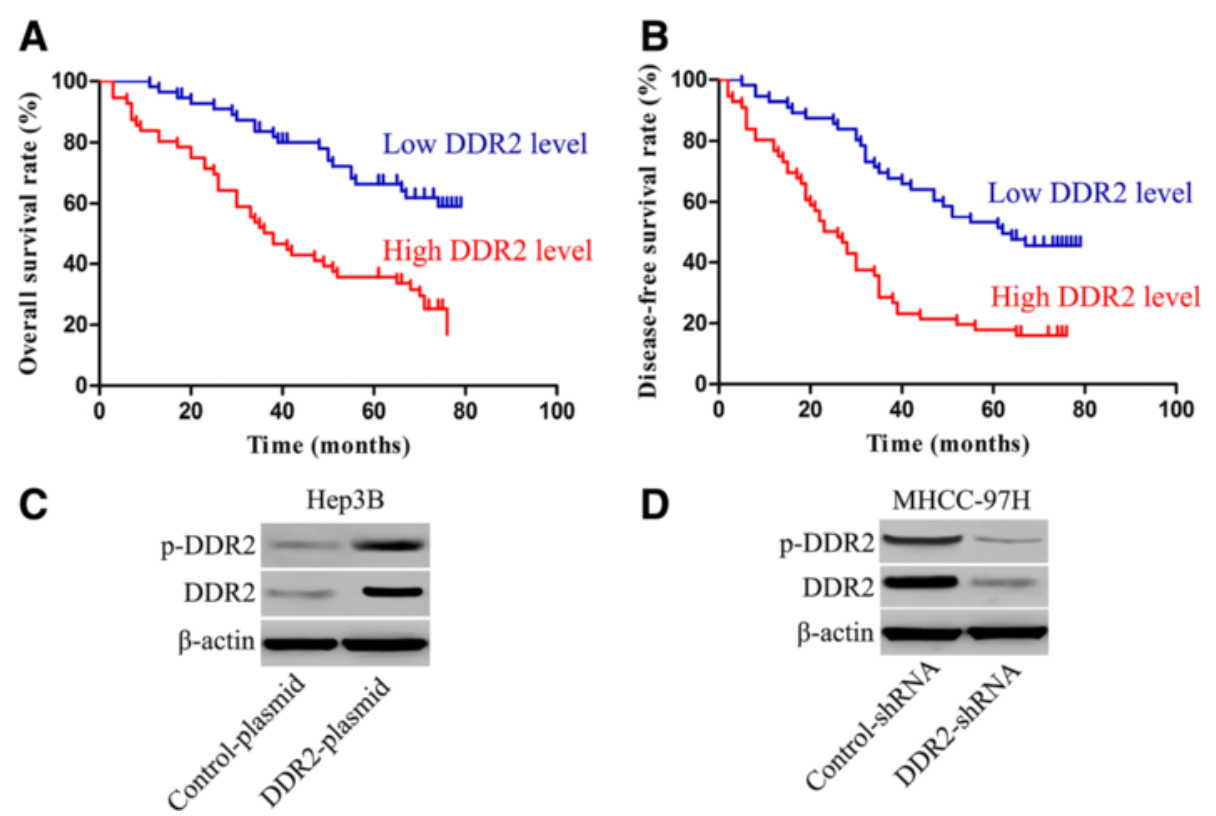

D

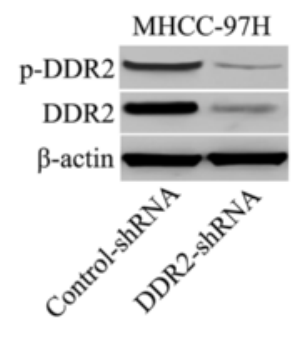

Fig. 2 Prognostic value of DDR2 protein expression in HCC and its correlation with phosphorylated DDR2 (p-DDR2) in HCC cells. a Overall survival for DDR2 protein expression (log-rank, $P<0.01$ ). b Disease-free survival for DDR2 protein expression (log-rank, $P<0.01$ ). $\mathbf{c}$ Immunoblotting analysis of DDR2 and p-DDR2 in Hep3B cells with DDR2 expressing plasmid transfection or control plasmid transfection. $\mathbf{d}$ Immunoblotting analysis of DDR2 and p-DDR2 in MHCC-97H cells with DDR2-shRNA transfection or control-shRNA transfection 
Table 3 Univariate prognostic analysis of overall survival and disease-free survival in HCC patients

\begin{tabular}{|c|c|c|c|c|c|c|c|}
\hline \multirow[t]{2}{*}{ Variable } & \multirow[t]{2}{*}{$n$} & \multicolumn{3}{|c|}{ Overall survival rate (\%) } & \multicolumn{3}{|c|}{ Disease-free survival rate (\%) } \\
\hline & & $3 y$ & $5 y$ & $P$ value & $3 y$ & $5 y$ & $P$ value \\
\hline \multicolumn{8}{|l|}{ Gender } \\
\hline Female & 25 & 87.7 & 66.8 & & 56.0 & 44.0 & \\
\hline Male & 87 & 60.9 & 46.4 & 0.100 & 47.1 & 31.8 & 0.234 \\
\hline \multicolumn{8}{|l|}{ Age (year) } \\
\hline$\leq 45$ & 29 & 82.4 & 57.3 & & 62.1 & 37.9 & \\
\hline$>45$ & 83 & 61.4 & 40.8 & 0.417 & 44.6 & 34.7 & 0.639 \\
\hline \multicolumn{8}{|l|}{ HBsAg status ${ }^{a}$} \\
\hline Negative & 22 & 90.5 & 85.4 & & 72.7 & 59.1 & \\
\hline Positive & 90 & 61.1 & 41.4 & $0.008^{*}$ & 43.3 & 29.6 & $0.017^{*}$ \\
\hline \multicolumn{8}{|l|}{ Cirrhosis } \\
\hline No & 37 & 64.2 & 58.1 & & 51.4 & 39.9 & \\
\hline Yes & 75 & 68.0 & 47.7 & 0.440 & 48.0 & 33.3 & 0.457 \\
\hline \multicolumn{8}{|l|}{$\operatorname{AFP}\left(\mu \mathrm{g} / \mathrm{l}^{\mathrm{b}}\right.$} \\
\hline$\leq 400$ & 55 & 61.3 & 53.4 & & 49.1 & 39.7 & \\
\hline$>400$ & 57 & 71.9 & 48.7 & 0.699 & 49.1 & 31.6 & 0.714 \\
\hline \multicolumn{8}{|l|}{ Tumor size } \\
\hline$\leq 5 \mathrm{~cm}$ & 37 & 75.7 & 58.9 & & 51.4 & 39.9 & \\
\hline$>5 \mathrm{~cm}$ & 75 & 62.3 & 46.8 & 0.398 & 48.0 & 33.3 & 0.938 \\
\hline \multicolumn{8}{|l|}{ Tumor number } \\
\hline Single & 86 & 71.8 & 57.1 & & 55.8 & 43.9 & \\
\hline Multiple & 26 & 50.0 & 30.8 & $<0.001^{*}$ & 26.9 & 7.7 & $<0.001^{*}$ \\
\hline \multicolumn{8}{|l|}{ Tumor capsule } \\
\hline Complete & 20 & 75.0 & 52.2 & & 50.0 & 44.4 & \\
\hline Incomplete & 92 & 65.0 & 50.5 & 0.507 & 48.9 & 33.7 & 0.611 \\
\hline \multicolumn{8}{|c|}{ Vascular invasion } \\
\hline No & 86 & 67.2 & 55.0 & & 53.5 & 41.6 & \\
\hline Yes & 26 & 65.4 & 37.1 & $0.011^{*}$ & 34.6 & 15.4 & $0.004^{*}$ \\
\hline \multicolumn{8}{|c|}{ Edmondson grade } \\
\hline$|/| \mid$ & 64 & 76.3 & 56.0 & & 64.1 & 44.8 & \\
\hline III/IV & 48 & 54.2 & 43.7 & $0.027^{*}$ & 29.2 & 22.9 & $0.002^{*}$ \\
\hline \multicolumn{8}{|l|}{ TNM stage } \\
\hline$|/| \mid$ & 67 & 69.8 & 62.0 & & 58.2 & 47.5 & \\
\hline III/IV & 45 & 62.2 & 34.0 & $<0.001^{*}$ & 35.6 & 17.8 & $0.001^{*}$ \\
\hline \multicolumn{8}{|l|}{ DDR2 level } \\
\hline Low & 56 & 83.7 & 66.3 & & 69.6 & 53.1 & \\
\hline High & 56 & 50.0 & 35.7 & $<0.001^{*}$ & 28.6 & 17.9 & $<0.001^{*}$ \\
\hline
\end{tabular}

(HR 2.051; $95 \%$ CI, 1.228-3.425; $P<0.05$ ) (Table 4). These data indicates that the DDR2 expression level is an independent prognosis factors of HCC patients.

Overexpression of DDR2 is associated with DDR2 activation To explore whether DDR2 overexpression is associated with DDR2 activation, we detected the phosphorylated
DDR2 (p-DDR2) expression in HCC cells, which was previously shown to be associated with DDR2 activation in several studies. As expected, overexpression of DDR2 evidently increased the level of p-DDR2 in Hep3B cells (Fig. 2c). Furthermore, knockdown of DDR2 expression markedly attenuated the phosphorylation of DDR2 in MHCC-97H cells (Fig. 2d). Our 
Table 4 Multivariate analysis of factors contributing to overall survival and disease-free survival in HCC patients

\begin{tabular}{|c|c|c|c|c|}
\hline \multirow[t]{2}{*}{ Variable } & \multicolumn{2}{|l|}{ Overall survival rate } & \multicolumn{2}{|c|}{ Disease-free survival rate } \\
\hline & $\mathrm{HR}(95 \% \mathrm{Cl})^{\mathrm{a}}$ & $\overline{P \text { value }}$ & $\mathrm{HR}(95 \% \mathrm{Cl})^{\mathrm{a}}$ & $P$ value \\
\hline HBsAg status ${ }^{b}$ & $3.021(1.335-6.836)$ & $0.008^{*}$ & $2.556(1.300-5.026)$ & $0.007^{*}$ \\
\hline Tumor number & $2.374(1.117-5.045)$ & $0.025^{*}$ & $2.758(1.363-5.579)$ & $0.005^{*}$ \\
\hline Vascular invasion & $0.912(0.451-1.847)$ & 0.799 & $1.086(0.553-2.136)$ & 0.810 \\
\hline TNM stage & $1.269(0.579-2.784)$ & 0.552 & $0.950(0.463-1.953)$ & 0.890 \\
\hline Edmondson grade & 0.999 (0.574-1.739) & 0.998 & $1.389(0.854-2.260)$ & 0.185 \\
\hline DDR2 level & $2.169(1.198-3.927)$ & $0.011^{*}$ & $2.051(1.228-3.425)$ & $0.006^{*}$ \\
\hline
\end{tabular}

${ }^{\mathrm{a}} \mathrm{HR}$ hazard ratio, ${ }^{\mathrm{b}} \mathrm{Hbs} \mathrm{Ag}$ hepatitis B surface antigen, $\mathrm{Cl}$ confidence interval, ${ }^{*} P<0.05$

results indicate that DDR2 amplification is associated with DDR2 activation.

\section{DDR2 promotes EMT and enhances invasion and migration ability in HCC}

We up-regulated DDR2 expression in Hep3B cells by DDR2 expressing plasmid. Transwell assays were performed to detect the effect of altering DDR2 expression levels on HCC cell invasion and migration. In our study, we found that up-regulation of DDR2 expression obviously promoted HCC cell migration and invasion $(P<0.01$, respectively, Fig. 3a). Next, we down-regulated DDR2 expression by DDR2-shRNA vectors in MHCC-97H cells. As expected, silencing DDR2 was found to inhibit MHCC-97H cell migration and invasion by Transwell assays $(P<0.05$, respectively, Fig. 3b). Moreover, DDR2 overexpression-induced EMT was associated with reduced E-cadherin expression and elevated Vimentin expression in Hep3B cells $(P<0.01$, respectively, Fig. 3c). Otherwise, silencing DDR2-induced suppression of EMT in MHCC97H cells was associated with elevated E-cadherin expression and reduced Vimentin expression. Our data indicate that DDR2 expression is associated with EMT phenotype and tumor cell migration and invasion in $\operatorname{HCC}(P<0.01$, respectively, Fig. 3d).

\section{DDR2 facilitates EMT via activating ERK signaling and stabilizing SNAIL1}

To explore the underlying mechanism by which DDR2 regulates cell migration, invasion and EMT in $\mathrm{HCC}$, we detected the effect of DDR2 on several transcription factors considered to be inducers of EMT, such as SNAIL1, TWIST1 and ZEB1. We found that up-regulation of DDR2 in Hep3B cells increased the SNAIL1 protein expression and knockdown of DDR2 in MHCC-97H cells resulted in a decreased level of SNAIL1 protein. However, overexpression or depletion of DDR2 did not alter the expression of TWIST1 and ZEB1. (Fig. 4a-b). Notably, overexpression or depletion of DDR2 did not alter SNAIL1 mRNA expression (Fig. 4c-d). Considering the fact that DDR2 controls the SNAIL1 expression posttranslationally, cycloheximide chase experiments were performed to detect the half-life of SNAIL1. As expected, DDR2 overexpression in Hep3B cells obviously increased the half-life of SNAIL1 and DDR2 depletion in MHCC-97H cells markedly decreased the half-life of SNAIL1 (Fig. 4e-f).

Next, expression of DDR2 and SNAIL1 was assessed by immunohistochemistry in HCC and matched tumoradjacent tissues. Both DDR2 and SNAIL1 levels were found to be evidently higher in HCC samples as compared with those in corresponding adjacent no tumorous samples (Fig. 5a-b). And there was indeed an evident positive correlation between the expression of DDR2 and SNAIL1 $(r=0.605, P<0.01$, Spearman's correlation test) (Fig. 5c-d). To further figure out the role of DDR2 in the stabilization of SNAIL1 in HCC cells, we examined whether DDR2 directly interacted with SNAIL1 using co-immunoprecipitation assay. However, the coimmunoprecipitation measurement revealed that DDR2 protein was not bound with SNAIL1 protein directly in MHCC-97H cells (data not shown).

Since ERK activation was recently shown to be critical for SNAIL1 stabilization and EMT induction, we investigated the ERK activation and its correlation with DDR2, SNAIL1 and EMT in HCC cells [29]. We found that overexpression of DDR2 in Hep3B cells increased the expression of p-ERK2, SNAIL1 and Vimentin and decreased the E-cadherin expression (Fig. 6a). DDR2 depletion in $\mathrm{MHCC}-97 \mathrm{H}$ cells resulted in a down-regulation of p-ERK2, SNAIL1 and Vimentin and an up-regulation of E-cadherin (Fig. 6b). ERK2 depletion in MHCC-97H cells attenuated the expression of SNAIL1, and Vimentin and increased the E-cadherin expression. (Fig. 6b). SNAIL1 depletion in MHCC-97H cells decreased the Vimentin expression and increased the E-cadherin expression (Fig. 6b). To further confirm the role of ERK2 in DDR2induced SNAIL1 stabilization in HCC cells, we examined whether ERK2 directly interacted with SNAIL1 using co-immunoprecipitation assay. As expected, the coimmunoprecipitation measurement revealed that ERK2 protein was bound with SNAIL1 protein directly in MHCC-97H cells (Fig. 6c). Thus, these data strongly suggest that DDR2 regulates SNAIL1 stability via stimulating 


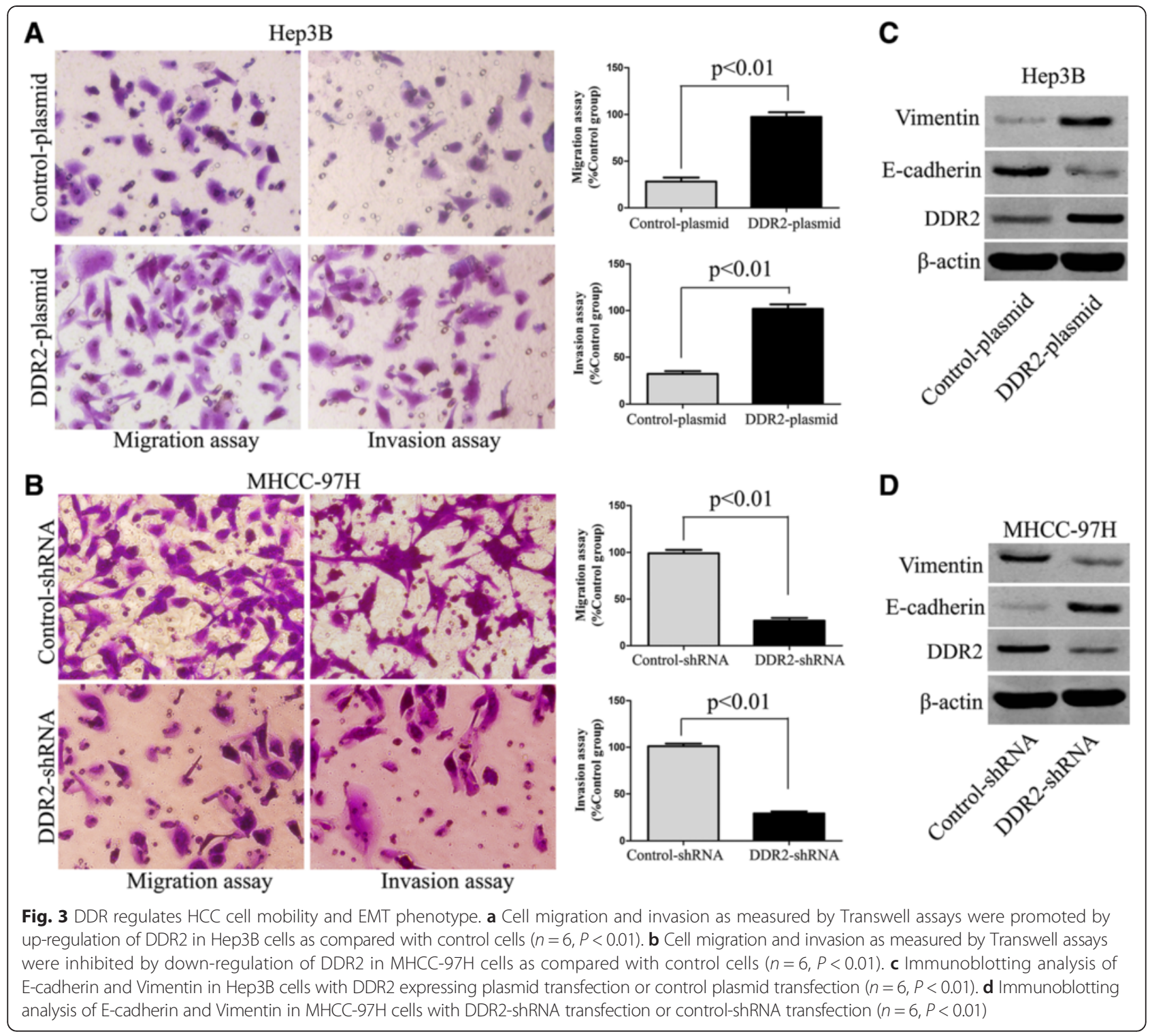

ERK2 activity and DDR2 may function as a key regulatory factor in HCC cell migration, invasion and EMT.

\section{DDR2 up-regulates MT1-MMP and MMP2 expression through ERK2/ SNAIL1 signaling in HCC}

It is well known that DDR2 can regulate metalloproteinase activity in stellate cells [12]. And DDR2 expression has been associated with increased MMPs expression $[13,30]$. To better understand the downstream molecules involved in DDR2 signaling-induced HCC migration and invasion, we identified whether DDR2 affected MT1-MMP and MMP2 expression in HCC cells. In our study, we found that up-regulation of DDR2 expression obviously increased MT1-MMP and MMP2 expression in Hep3B cells (Fig. 6d). In contrary, knockdown of DDR2 expression markedly decreased the MT1-MMP and
MMP2 expression in MHCC-97H cells (Fig. 6e). Furthermore, we found that DDR2 up-regulated MT1-MMP and MMP2 expression through ERK2/SNAIL1 signaling in HCC cells (Fig. 6e).

\section{Collagen I induces DDR2/ERK2/SNAIL1 signaling activation in $\mathrm{HCC}$}

Considering the fact that DDR2 belongs to RTK family and type I collagen is the DDR2 ligand, we examined the effect of collagen I on the DDR2 phosphorylation. In our study, exposure of HCC cells to collagen I resulted in tyrosine phosphorylation and activation of DDR2 (Fig. 6f). Recently, it was shown that collagen I can activate DDR2/ ERK2/SNAIL1 signaling axis in breast cancer. To better understand the underlying mechanism of DDR2 in HCC, we further determined whether collagen I affected DDR2/ 


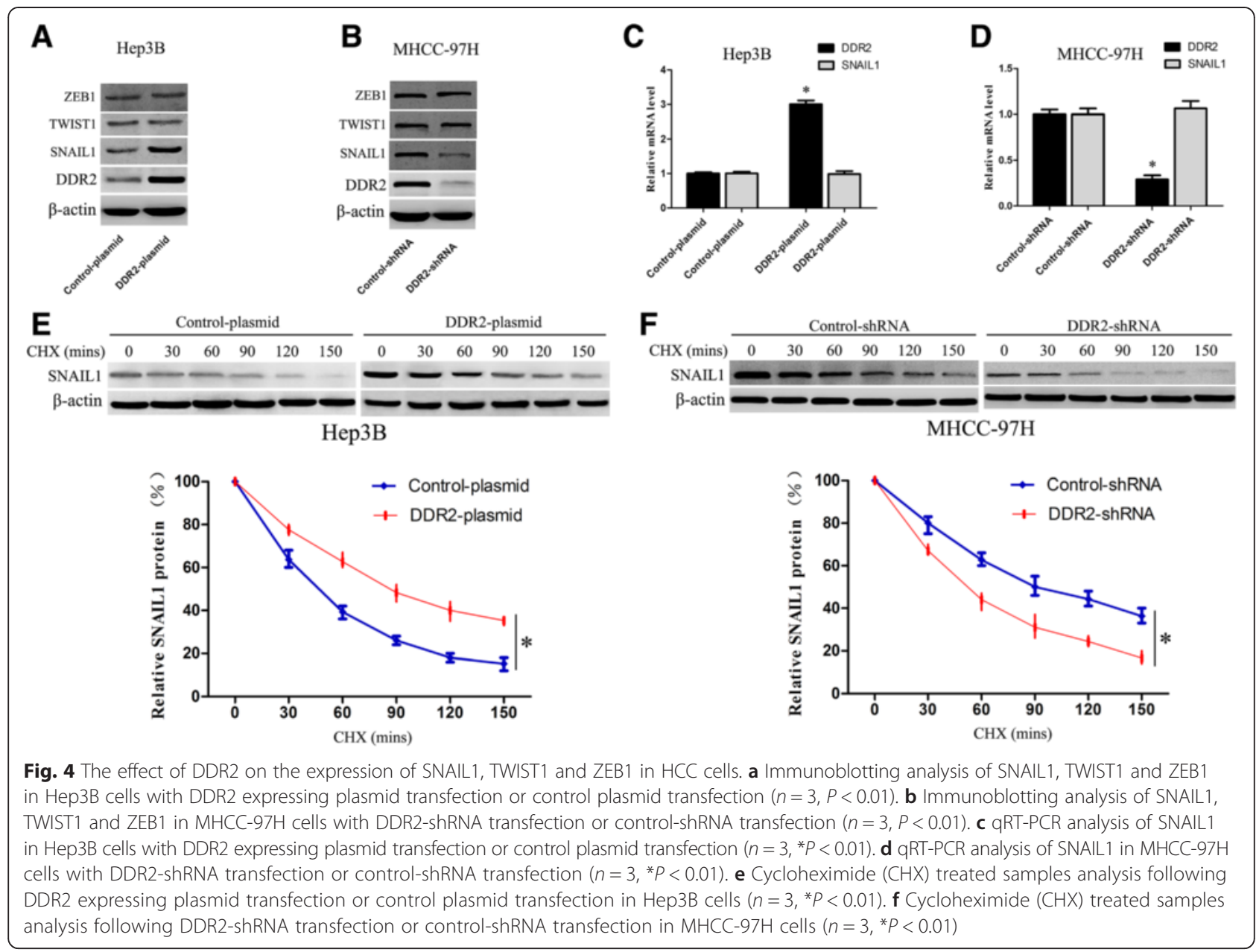

ERK2/SNAIL1 signaling activation in HCC cells. In this study, we found that collagen I significantly activates DDR2/ERK2/SNAIL1 signaling axis in HCC (Fig. 6f).

\section{Discussion}

DDR2, a receptor tyrosine kinase, is considered to be involved in the progression of many cancer types [16, 23]. Increasing evidences have demonstrated that up-regulation of DDR2 is commonly observed in multiply tumor types including breast and prostate cancer [18, 15]. Moreover, several researches revealed that activated DDR2 could up-regulate the expression level of MMP-1, MMP-2 and MMP-13 [12, 13, 30].

Here we revealed that DDR2 expression in HCC cell lines was obviously higher than that in immortalized non-tumourigenic hepatocyte cell line L02. Our findings also showed that DDR2 expression was significantly higher in HCC tissues compared with matched normal tumoradjacent tissues. Furthermore, high DDR2 level was evidently correlated with tumor number, vascular invasion, Edmondson-Steiner grade and TNM stage in HCC. Importantly, our results indicates that overexpression of DDR2 was obviously correlated with shorter OS and DFS, which is an assumed molecular marker for poor prognosis in HCC. Additionally, Cox repression analysis confirmed that DDR2 is a novel independent factor for HCC. Taken together, our results demonstrated that DDR2 overexpression may promote tumor progression and is a critical factor for prognosis determination in HCC patients.

In this study, we detected the phosphorylated DDR2 expression level (p-DDR2), which was previously shown to be associated with DDR2 activation. Our data indicate that DDR2 overexpression is related to the increase in activated p-DDR2. In our study, we also investigated the role of DDR2 in HCC cells. We found that up-regulation of DDR2 obviously promoted cell migration and invasion in Hep3B cells. In contrast, DDR2 depletion significantly inhibited cell migration and invasion in MHCC-97H cells. Our data suggest that DDR2 indeed facilitates the migration and invasion of HCC cell. EMT, a dynamic biological process, is crucial in the development of invasiveness and metastatic potential of many cancers including HCC [29]. The enhanced EMT process in tumor cells has been 


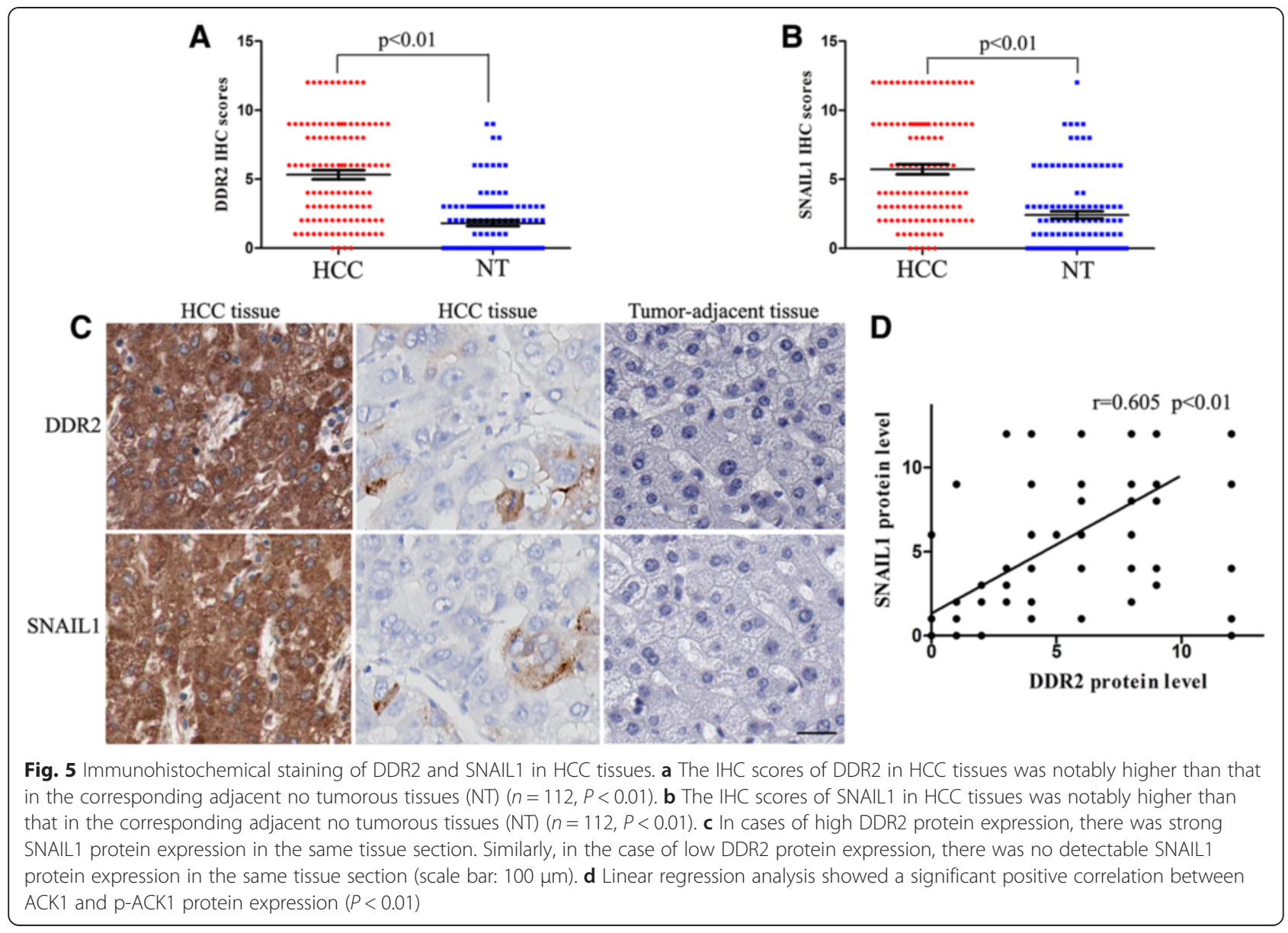

shown to increase the risk of invasion and metastasis and is associated with negative prognosis in patients with HCC [31]. Thus, we detected the expression of epithelial biomarker, E-cadherin, and mesenchymal biomarker, Vimentin with altering DDR2 expression in HCC cells. Notably, DDR2 overexpression markedly facilitates EMT and is associated with reduced E-cadherin level and elevated Vimentin level. Meanwhile, DDR2 depletion significantly inhibits EMT and is associated with elevated E-cadherin level and reduced Vimentin level. Our results revealed that DDR2 may promote HCC cell migration and invasion via inducing EMT.

Many signals generated within the cancer microenvironment activate transcription factors considered to be promoters of EMT, such as SNAIL1, TWIST1 and ZEB1 $[23,32]$. Here we detected the effect of DDR2 on these transcription factors in HCC cells. Overexpression or depletion of DDR2 affected the protein expression of SNAIL1, whereas the level of TWIST1 and ZEB1 was unchanged. Notably, overexpression or depletion of DDR2 did not alter SNAIL1 mRNA expression. Furthermore, there was indeed an evident positive correlation between the expression of DDR2 and SNAIL1 in HCC tissues. Interestingly, we demonstrated that DDR2 protein was not bound with SNAIL1 protein directly in MHCC-97H cells. Our results revealed that SNAIL1 stabilization by DDR2 was unlikely to be the result of direct interaction by DDR2.

The ERK signaling is a ubiquitous signal transduction pathway that regulates important cellular processes, such as proliferation, migration and invasion [29, 33]. Increasing evidences have demonstrated that the activation of ERK is believed to contribute to tumorigenesis in many cancer types including HCC [29, 24]. Notably, ERK activation was recently shown to be critical for SNAIL1 stabilization and EMT induction [23]. Here we investigated the ERK activation and its correlation with DDR2 and SNAIL1 in HCC cells. Overexpression or depletion of DDR2 affected p-ERK2 expression, whereas the level of p-ERK1 was unchanged. Moreover, ERK2 depletion attenuated the SNAIL1 level in HCC cells. Importantly, ERK2 protein was bound with SNAIL1 protein directly in HCC cells. Our data strongly suggest that DDR2 regulates SNAIL1 stability via stimulating ERK2 activity.

MMPs activity has been related with cancer cell migration and invasion [28]. As two members of MMPs, MT1-MMP and MMP2 are critical factors in the migration and invasion of HCC [34, 28]. Considering the 

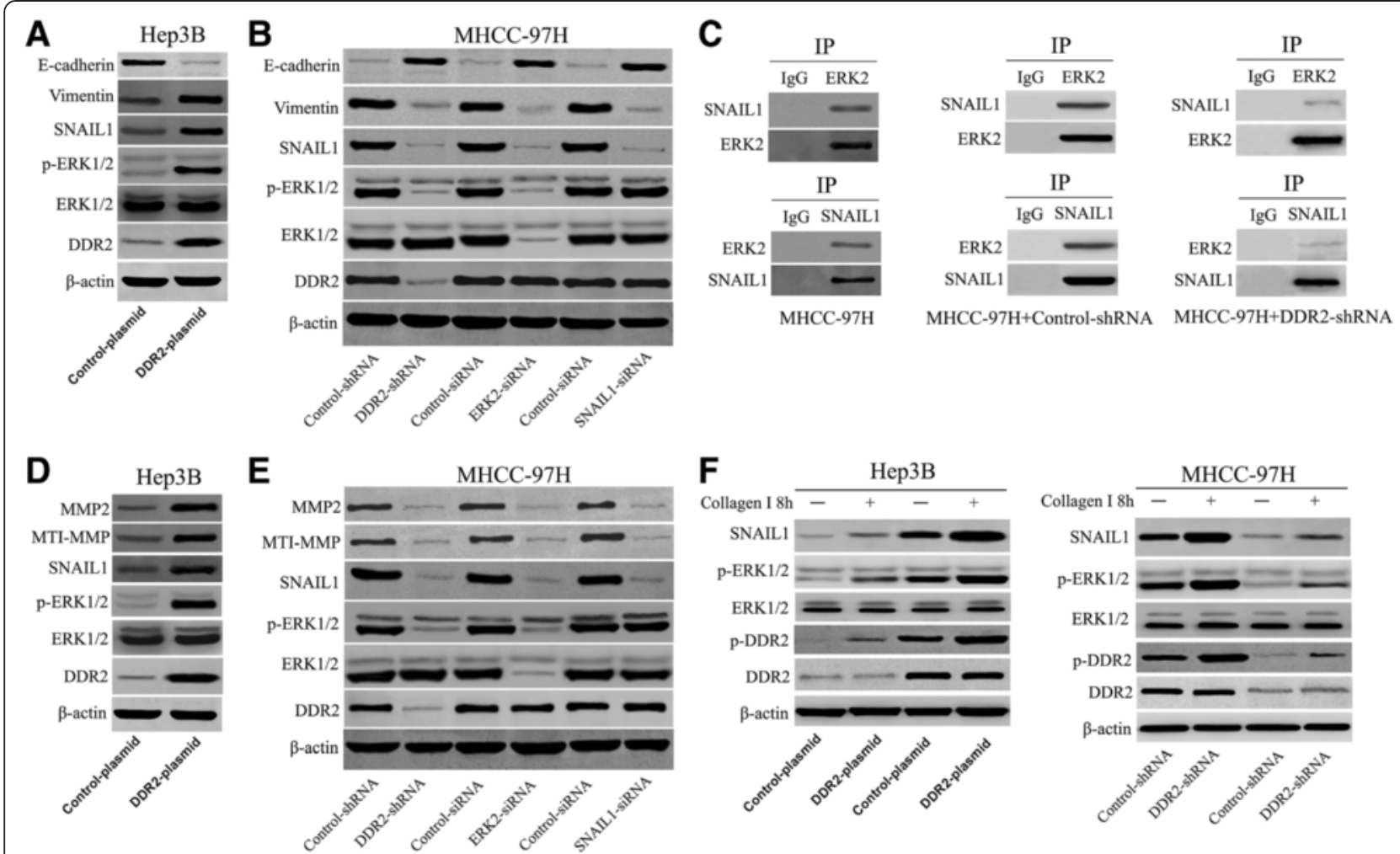

Fig. 6 DDR2 induces EMT and up-regulates the expression of MTI-MMP and MMP2 through ERK2/SNAIL1 signaling in HCC cells. a Hep3B cells that had been transfected with DDR2 expressing plasmid or control plasmid, respectively, were subjected to western blotting for ERK1/2, p-ERK1/2, SNAIL1, Vimentin and E-cadherin. b MHCC-97H cells that had been transfected with control-shRNA, DDR2-shRNA, control-siRNA, ERK2-siRNA, control-siRNA or SNAIL1-siRNA, respectively, were subjected to western blotting for DDR2, ERK1/2, p-ERK1/2, SNAIL1, Vimentin and E-cadherin). c Co-immunoprecipitation assays showed that ERK2 was bound with SNAIL1 directly. $\mathbf{d}$ Hep3B cells that had been transfected with DDR2 expressing plasmid or control plasmid, respectively, were subjected to western blotting for ERK1/2, p-ERK1/2, SNAIL1, MTI-MMP and MMP2. e MHCC-97H cells that had been transfected with control-shRNA, DDR2-shRNA, control-siRNA, ERK2-siRNA, control-siRNA or SNAIL1-siRNA, respectively, were subjected to western blotting for DDR2, ERK1/2, p-ERK1/2, SNAIL1, MTI-MMP and MMP2. f HCC cells that had been transfected with indicated plasmids were added to plates coated with collagen I ( $2 \mathrm{mg} / \mathrm{ml}$, BD Biosciences) for $8 \mathrm{~h}$ and western blotting were performed

fact that a key biological function of RTKs is to regulate the expression of MMPs, we identified whether DDR2 affected MT1-MMP and MMP2 expression in HCC cells $[12,13]$. We found that up-regulation of DDR2 expression obviously increased MT1-MMP and MMP2 expression and DDR2 depletion markedly decreased the MT1-MMP and MMP2 expression. Furthermore, DDR2 up-regulates MT1-MMP and MMP2 expression through ERK2/SNAIL1 signaling in HCC cells. These data suggest that DDR2-induced ERK2/SNAIL1 signaling activation may be responsible for the progression of EMT and up-regulation of MT1-MMP and MMP2 expression, which in turn facilitates migration and invasion of HCC.

DDR2 belongs to RTK family and type I collagen is the DDR2 ligand. Recently, it was shown that the type I collagen can activate DDR2/ERK2/SNAIL1 signaling axis in breast cancer. In our study, we found that collagen I can activate DDR2/ERK2/SNAIL1 signaling axis in HCC cells. Our results are consistent with previous findings on breast cancer, suggesting the type I collagen can cause DDR2 phosphorylation and induce DDR2/ERK2/SNAIL1 signaling axis hyperactivation.

In summary, we found that DDR2 expression level is up-regulated in HCC as compared with non-cancerous liver samples and that overexpression of DDR2 is correlated with clinicopathological characteristics of poor prognosis in HCC. Additionally, we found that DDR2 level is an independent factor for predicting the overall survival and disease-free survival of patients with HCC. Notably, DDR2 can facilitate cell invasion, migration and epithelial-mesenchymal transition via activating ERK signaling and stabilizing SNAIL1 and up-regulate MT1MMP and MMP2 expression through ERK2/SNAIL1 signaling in HCC.

\section{Conclusions}

Our study provides a better understanding on both the molecular mechanism and functional role of DDR2 in human HCC. Our current work revealed that DDR2 has 
an oncogenic role in hepatocarcinogenesis by facilitating cancer cell invasion, migration and EMT via activating ERK2 and stabilizing SNAIL1. Notably, DDR2 can upregulate MT1-MMP and MMP2 expression through ERK2/SNAIL1 signaling in HCC. Our study identified that DDR2 is a novel regulator of EMT via DDR2induced ERK2/SNAIL1 signaling activation, indicating its potential therapeutic value for reducing $\mathrm{HCC}$ invasion and metastasis.

\section{Competing interests}

The authors declare that they have no competing interests.

\section{Authors' contributions}

$\mathrm{HL}$ and GT were responsible for the design of research. BX and WL conducted the experiments and drafted the manuscript. BZ and SX participated in the data analysis. JY and XW helped in sample collection. And the authors declare no conflict of interest. All authors read and approved the final manuscript.

\section{Acknowledgements \\ This study was supported by grants from the National Natural Science Foundation of China (No. 30600156), the Natural Science Foundation of Guangdong Province (No.2014A030313090 and No. 2014A030313190), the Science and Technology Projects Foundation of Jiangxi Province (No. 20151BBG70085) and the Science and Technology Projects Foundation of Guangzhou City (No. 1515000250).}

\section{Author details \\ 'Department of Hepatobiliary Surgery, The First Affiliated Hospital of Gannan Medical University, Ganzhou 341000, China. ²Department of General Surgery, The First Affiliated Hospital of Sun Yat-sen University, Guangzhou 510080, China. ${ }^{3}$ Department of Anesthesiology, The First Affiliated Hospital of Gannan Medical University, Ganzhou 341000, China. ${ }^{4}$ Department of Medical Imaging, The First Affiliated Hospital of Sun Yat-sen University, Guangzhou 510080, China. ${ }^{5}$ Department of Biochemistry, University of Leicester, Leicester LE1 $9 \mathrm{HN}, \mathrm{UK}$.}

Received: 23 June 2015 Accepted: 4 September 2015 Published online: 11 September 2015

\section{References}

1. Jemal A, Bray F, Center MM, Ferlay J, Ward E, Forman D. Global cancer statistics. CA Cancer J Clin. 2011;61(2):69-90. doi:10.3322/caac.20107.

2. Lafaro KJ, Demirjian AN, Pawlik TM. Epidemiology of hepatocellular carcinoma. Surg Oncol Clin N Am. 2015;24(1):1-17. doi:10.1016/j.soc.2014.09.001.

3. Liu Q, Yang P, Tu K, Zhang H, Zheng X, Yao Y, et al. TPX2 knockdown suppressed hepatocellular carcinoma cell invasion via inactivating AKT signaling and inhibiting MMP2 and MMP9 expression. Chin J Cancer Res. 2014;26(4):410-7. doi:10.3978/j.issn.1000-9604.2014.08.01.

4. Altekruse SF, McGlynn KA, Reichman ME. Hepatocellular carcinoma incidence, mortality, and survival trends in the United States from 1975 to 2005. J Clin Oncol. 2009;27(9):1485-91. doi:10.1200/jco.2008.20.7753.

5. Bosetti C, Levi F, Boffetta P, Lucchini F, Negri E, La Vecchia C. Trends in mortality from hepatocellular carcinoma in Europe, 1980-2004. Hepatology. 2008;48(1):137-45. doi:10.1002/hep.22312.

6. He F, Li J, Xu J, Zhang S, Xu Y, Zhao W, et al. Decreased expression of ARID1A associates with poor prognosis and promotes metastases of hepatocellular carcinoma. J Exp Clin Cancer Res. 2015;34:47. doi:10.1186/s13046-015-0164-3.

7. Bosetti C, Turati F, La Vecchia C. Hepatocellular carcinoma epidemiology. Best Pract Res Clin Gastroenterol. 2014;28(5):753-70. doi:10.1016/j.bpg.2014.08.007.

8. Liao R, Sun J, Wu H, Yi Y, Wang JX, He HW, et al. High expression of IL-17 and IL-17RE associate with poor prognosis of hepatocellular carcinoma. J Exp Clin Cancer Res. 2013;32:3. doi:10.1186/1756-9966-32-3.

9. Vogel W, Gish GD, Alves F, Pawson T. The discoidin domain receptor tyrosine kinases are activated by collagen. Mol Cell. 1997:1(1):13-23.

10. Shrivastava A, Radziejewski C, Campbell E, Kovac L, McGlynn M, Ryan TE, et al. An orphan receptor tyrosine kinase family whose members serve as nonintegrin collagen receptors. Mol Cell. 1997;1(1):25-34.
11. Leitinger B. Transmembrane collagen receptors. Annu Rev Cell Dev Biol. 2011;27:265-90. doi:10.1146/annurev-cellbio-092910-154013.

12. Olaso E, Ikeda K, Eng FJ, Xu L, Wang LH, Lin HC, et al. DDR2 receptor promotes MMP-2-mediated proliferation and invasion by hepatic stellate cells. J Clin Invest. 2001;108(9):1369-78. doi:10.1172/jci12373.

13. Olaso E, Labrador JP, Wang L, Ikeda K, Eng FJ, Klein R, et al. Discoidin domain receptor 2 regulates fibroblast proliferation and migration through the extracellular matrix in association with transcriptional activation of matrix metalloproteinase-2. J Biol Chem. 2002;277(5):3606-13. doi:10.1074/jbc.M107571200.

14. Chua HH, Yeh TH, Wang YP, Huang YT, Sheen TS, Lo YC, et al. Upregulation of discoidin domain receptor 2 in nasopharyngeal carcinoma. Head Neck. 2008;30(4):427-36. doi:10.1002/hed.20724.

15. Yan Z, Jin S, Wei Z, Huilian H, Zhanhai $Y$, Yue $T$, et al. Discoidin domain receptor 2 facilitates prostate cancer bone metastasis via regulating parathyroid hormone-related protein. Biochim Biophys Acta. 2014;1842(9):1350-63. doi:10.1016/j.bbadis.2014.04.018.

16. Xu J, Lu W, Zhang S, Zhu C, Ren T, Zhu T, et al. Overexpression of DDR2 contributes to cell invasion and migration in head and neck squamous cell carcinoma. Cancer Biol Ther. 2014;15(5):612-22. doi:10.4161/cbt.28181.

17. Kim D, Ko P, You E, Rhee $\mathrm{S}$. The intracellular juxtamembrane domain of discoidin domain receptor 2 (DDR2) is essential for receptor activation and DDR2-mediated cancer progression. Int J Cancer. 2014;135(11):2547-57. doi:10.1002/ijc.28901.

18. Ren T, Zhang J, Zhang J, Liu X, Yao L. Increased expression of discoidin domain receptor 2 (DDR2): a novel independent prognostic marker of worse outcome in breast cancer patients. Med Oncol. 2013;30(1):397. doi:10.1007/s12032-012-0397-3.

19. Tran DD, Corsa CA, Biswas H, Aft RL, Longmore GD. Temporal and spatial cooperation of Snail1 and Twist1 during epithelial-mesenchymal transition predicts for human breast cancer recurrence. Mol Cancer Res. 2011;9(12):1644-57. doi:10.1158/1541-7786.mcr-11-0371.

20. Kaufhold S, Bonavida B. Central role of Snail1 in the regulation of EMT and resistance in cancer: a target for therapeutic intervention. J Exp Clin Cancer Res. 2014;33:62. doi:10.1186/s13046-014-0062-0.

21. Liu Z, Tu K, Liu Q. Effects of microRNA-30a on migration, invasion and prognosis of hepatocellular carcinoma. FEBS Lett. 2014;588(17):3089-97. doi:10.1016/j.febslet.2014.06.037.

22. Drasin DJ, Robin TP, Ford HL. Breast cancer epithelial-to-mesenchymal transition: examining the functional consequences of plasticity. Breast Cancer Res. 2011;13(6):226. doi:10.1186/bcr3037.

23. Zhang K, Corsa CA, Ponik SM, Prior JL, Piwnica-Worms D, Eliceiri KW, et al. The collagen receptor discoidin domain receptor 2 stabilizes SNAIL1 to facilitate breast cancer metastasis. Nat Cell Biol. 2013;15(6):677-87. doi:10.1038/ncb2743.

24. Whittaker S, Marais R, Zhu AX. The role of signaling pathways in the development and treatment of hepatocellular carcinoma. Oncogene. 2010;29(36):4989-5005. doi:10.1038/onc.2010.236.

25. Muntane J, De la Rosa AJ, Docobo F, Garcia-Carbonero R, Padillo FJ. Targeting tyrosine kinase receptors in hepatocellular carcinoma. Curr Cancer Drug Targets. 2013;13(3):300-12

26. van Zijl F, Zulehner G, Petz M, Schneller D, Kornauth C, Hau M, et al. Epithelial-mesenchymal transition in hepatocellular carcinoma. Future Oncol. 2009;5(8):1169-79. doi:10.2217/fon.09.91.

27. Zhang L, Huang G, Li X, Zhang Y, Jiang Y, Shen J, et al. Hypoxia induces epithelial-mesenchymal transition via activation of SNAI1 by hypoxiainducible factor -1alpha in hepatocellular carcinoma. BMC Cancer. 2013;13:108. doi:10.1186/1471-2407-13-108.

28. Liu Q, Tu K, Zhang H, Zheng X, Yao Y, Liu Q. TPX2 as a novel prognostic biomarker for hepatocellular carcinoma. Hepatol Res. 2014. doi:10.1111/hepr.12428.

29. Wang ZL, Fan ZQ, Jiang HD, Qu JM. Selective Cox-2 inhibitor celecoxib induces epithelial-mesenchymal transition in human lung cancer cells via activating MEK-ERK signaling. Carcinogenesis. 2013;34(3):638-46. doi:10.1093/carcin/bgs367.

30. Xu L, Peng H, Wu D, Hu K, Goldring MB, Olsen BR, et al. Activation of the discoidin domain receptor 2 induces expression of matrix metalloproteinase 13 associated with osteoarthritis in mice. J Biol Chem. 2005;280(1):548-55. doi:10.1074/jbc.M411036200.

31. Kan H, Guo W, Huang Y, Liu D. MicroRNA-520 g induces epithelialmesenchymal transition and promotes metastasis of hepatocellular 
carcinoma by targeting SMAD7. FEBS Lett. 2015;589(1):102-9. doi:10.1016/j.febslet.2014.11.031

32. Du R, Wu S, Lv X, Fang H, Wu S, Kang J. Overexpression of brachyury contributes to tumor metastasis by inducing epithelial-mesenchymal transition in hepatocellular carcinoma. J Exp Clin Cancer Res. 2014;33:105 doi:10.1186/s13046-014-0105-6.

33. Zheng F, Wu J, Zhao S, Luo Q, Tang Q, Yang L, et al. Baicalein increases the expression and reciprocal interplay of RUNX3 and FOXO3a through crosstalk of AMPKalpha and MEK/ERK1/2 signaling pathways in human non-small cell lung cancer cells. J Exp Clin Cancer Res. 2015;34:41. doi:10.1186/s13046-015-0160-7

34. Ip YC, Cheung ST, Leung KL, Fan ST. Mechanism of metastasis by membrane type 1-matrix metalloproteinase in hepatocellular carcinoma. World J Gastroenterol. 2005;11(40):6269-76.

\section{Submit your next manuscript to BioMed Central and take full advantage of:}

- Convenient online submission

- Thorough peer review

- No space constraints or color figure charges

- Immediate publication on acceptance

- Inclusion in PubMed, CAS, Scopus and Google Scholar

- Research which is freely available for redistribution 